\title{
Approach to Assessing Quality Indicators
}

\author{
O. B. Ivanets, \\ Department of Biocybernetics and Aerospace Medicine \\ National Aviation University \\ Kyiv, Ukraine; \\ olchik2104@ukr.net \\ Yu. M. Tereshchenko, \\ Department of Aviation Engines, \\ National Aviation University \\ Kyiv, Ukraine; \\ terj@nau.edu.ua
}

\author{
I. V. Morozova, \\ Department of Electronic, \\ National Aviation University \\ Kyiv, Ukraine; \\ iramoro@ukr.net
}

\begin{abstract}
The article analyzes the indicators obtained in the results of experimental research. These indicators determine physical, chemical, operational and environmental changes as a result of the influence of magnetic fields. A comparative analysis of electrophysical influence on liquid systems was carried out. Calculation of the uncertainty of input values during experimental studies allowed to increase the reliability of decision making regarding the degree of electro-physical impact on ecological indicators.
\end{abstract}

Keywords - environmental performance, engine, combine uncertainty, fuel volume, uncertainty of test results

\section{INTRODUCTION}

One of the key indicators of the quality of a thermal engine is its reliability. In many respects, the level of reliability when operating the engine depends on the potential properties laid down at the design and production stage. However, under real-life conditions, the pledged level of reliability of the thermal engine is not fully realized. Particularly acute is the problem of using exhaustible resources that are actively used in internal combustion engines [1].

Particular attention during the efficient use of the thermal engine is devoted to the performance of the fuel equipment, since the violations of the basic control parameters leads to a decrease in dynamic, power, environmental and economic performance, causing significant losses of fuel, as well as reduce the life of the engines. Thus, quality issues as the fuel itself so its use is crucial in determining the prospects for the development of engine construction.

\section{PROBLEM RESOLUTION}

Recently, prerequisites have been created for the industrial development of new methods and the most promising areas for improving engine performance. So, to change the physicochemical, operational and environmental properties of liquid systems, the method of electrophysical effects is widely studied. This method is based on the impact on the physico-chemical properties of hydrocarbon fuels in order to ensure reliable operation of heat engines, reduce fuel consumption and reduce toxicity of exhaust gases under operating conditions. The paper proposes a device based on the mechanism of magnetic impact on atomic particles of hydrocarbon fuels in order to assess the effectiveness of this impact on the performance properties of fuels and processes in the combustion chambers of heat engines[1]. The appearance of the device is shown in Fig.1.

The electrons of the atom and the nucleus of some elements $\left(H^{1}, C^{13}, O^{17}, P^{31}, L i^{7}, F^{19}\right.$, and a number of others) have magnetic and mechanical moments. The electron spin $I= \pm \frac{1}{2}$ manifests itself as a fine structure in the atomic spectra. In the case of nuclei, this property is found in the form of a hyperfine structure. The magnetic moment of the electron interacts with the magnetic moment of the nucleus. The interaction energy depends on the relative orientation of the spins or magnetic moments, and the number of possible orientations is determined by the spin of the nucleus. The total spin of the nucleus depends on whether the spins of the nuclear components compensate each other or not.

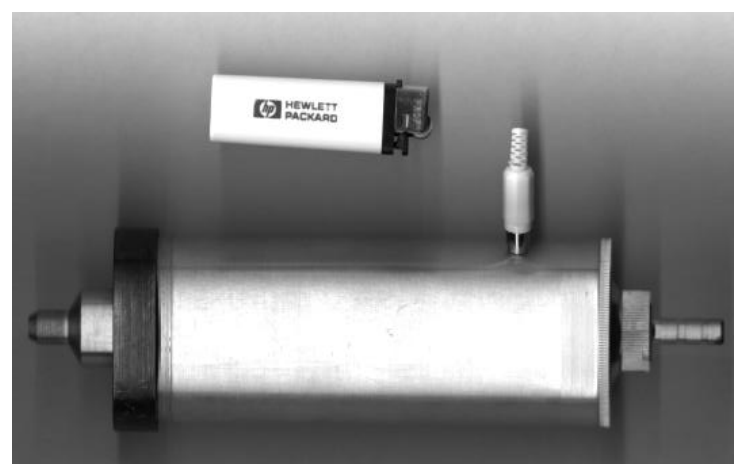

Fig. 1. Electrophysical impact device

In a magnetic field, the magnetic moments of a proton with a spin are oriented in only two ways - in the direction of the field or against the direction of the field. Each nucleus with a magnetic moment, falling into a magnetic field, acquires a zeeman additional energy $\mu H_{0}$. In this case, the hamiltonian has the form [2]:

$$
\mathcal{H}=-\mu_{n} H_{0},
$$

where $\mu_{n}$ is the magnetic moment of the proton, equal to $1.41 \cdot 10^{-26} \mathrm{~J} / \mathrm{T}$;

$H_{0}$ is the magnetic field strength, $\mathrm{A} / \mathrm{m}$.

Let the field $H_{0}$ be directed along the axis $Z$ of the coordinate system (Fig. 2).

Then the zeeman interaction is described by the hamiltonian:

$$
\mathcal{H}=-\gamma h H_{0} I_{0},
$$

When the total nuclear moment changes, its state changes significantly; its internal energy changes. From the principles 
of statistical physics, it follows that the ratio of the numbers of protons $\mathrm{n}$ with different orientations of magnetic moments is:

$$
\frac{n_{(+\mu)}}{n_{(-\mu)}}=e^{\frac{2 \mu H}{k T}}
$$

where $T$ is the absolute temperature of the liquid; $\left.n_{(+\mu)}, n_{(-\mu}\right)$ is the population levels; $k$ is the Boltzmann constant.

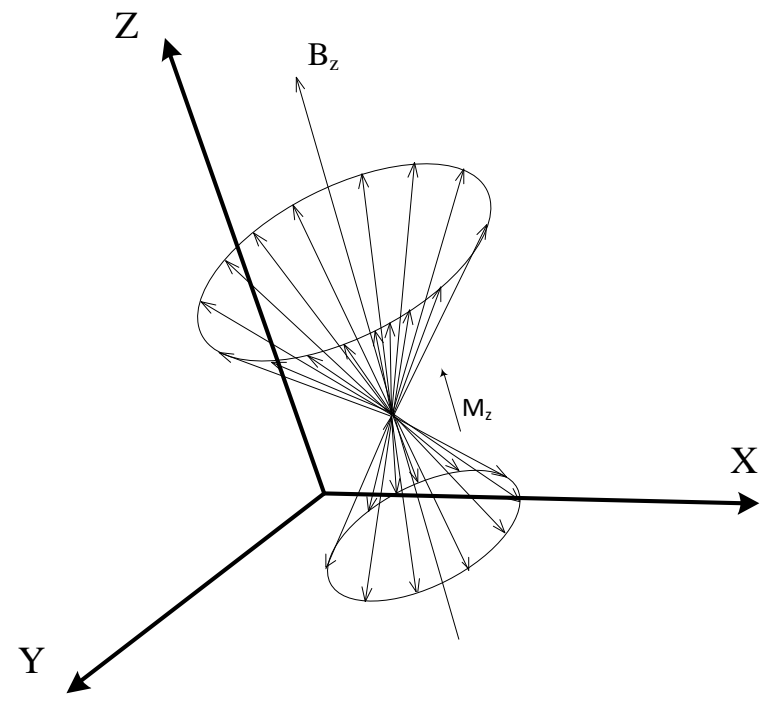

Fig. 2. Magnetic field strength directions

Under normal conditions, nuclear polarization $P$ is insignificant due to its smallness $\mu_{n}$ and is determined from the expression:

$$
P=\frac{n_{(+\mu)}-n_{(-\mu)}}{n_{(+\mu)}+n_{(-\mu)}}
$$

As the intensity of the external magnetic field increases, the difference in energy for the spin directed against the field and the spin directed along the field increases, which leads to an increase in polarization. However, this alignment of the spins does not occur immediately. Before the imposition of a magnetic field, the number of spins directed "up" and "down" was the same, and then in the field some of the spins directed against the magnetic field turned over.

The reorientation of the nuclear spin is accompanied by a change in its energy, so it can only occur in the presence of an object with which the nucleus exchanges energy. Such objects can be electrons, neighboring nuclei, paramagnetic elements (oxygen, etc.), ions, free radicals or a radio frequency circuit with a high Q-factor tuned to the frequency of precession of nuclei in an external magnetic field.

In the volume of the substance there is a total magnetic moment of the nuclei $M$ (magnetization) directed along the external field:

$$
M=\mu\left[n_{(+\mu)}-n_{(-\mu)}\right]
$$

Using expression (2.1), we can write:

$$
\begin{gathered}
\frac{n_{(+\mu)}-n_{(-\mu)}}{n_{(+\mu)}+n_{(-\mu)}}=\frac{e^{\frac{2 \mu H}{k T}}-1}{1+e^{\frac{2 \mu H}{k T}}}, \\
\frac{2 \mu H}{k T}<<1, \text { then } e^{\frac{2 \mu H}{k T}} \approx 1+\frac{2 \mu H}{k T}
\end{gathered}
$$

From where for the total number of nuclei in a unit volume of a substance $n_{0}$, the magnetic moment $\mathrm{M}$ is equal to:

$$
M=\frac{\mu^{2} H_{o} n_{o}}{k T}
$$

The sharp decrease in the intensity of the magnetic field leads to the fact that the additional energy of the proton, due to the ultra-thin interaction, is absorbed near the hydrogen by atomic elements of the non-zero electron spin. These elements can be paramagnetic oxygen, free radicals, etc., that is, paramagnetic centers interact with the basic chemical compound. An increase in the polarization of hydrogen nuclei when the magnetic field intensity changes will inevitably cause the destruction of electron shells in the molecules due to ultrafine interaction, which, in turn, causes induction currents that affect the physical properties of liquids.

Electrophysical method based on resonance energy absorption by the proton fluid system is proposed for increasing the efficiency, stability and universality of magnetic effects on liquid systems.

The method consists of simultaneously influencing the liquid of an inhomogeneous constant magnetic field and a resonant high-frequency electromagnetic field. In this case, the frequency of oscillations coincides with the frequency of precession of nuclei in a given magnetic field and there is a selective absorption of the energy of the generator at a frequency:

$$
\omega=\gamma H_{o},
$$

where $\gamma$ is the gyromagnetic ratio of the proton.

The alternating electromagnetic $H_{1}$ field perturbs the system of nuclear spins, which is described by the hamiltonian:

$\mathcal{H}^{\prime}(t)=\mathcal{H} \cos \omega t=q_{n} \beta\left(J_{x} H_{1 x}+J_{y} H_{1 y}+J_{z} H_{1 z}\right) \cos \omega t$

The influence of the electromagnetic field $H_{1}$ causes intense transitions from the "bottom to top" and, conversely, between the energy levels and the boltzmann distribution is violated. In this case, the electromagnetic energy of the field $H_{1}$ is partially absorbed by the proton liquid system, an absorption signal is observed, and, also, the energy of the medium increases. The power absorbed $P$ by the spin from the alternating electromagnetic field is:

$$
P=\hbar \omega W_{\hbar}=\hbar \omega n_{0} \frac{W}{1+2 W T_{1}}
$$


Thus, the resonant absorption of the proton fluid system by the electromagnetic energy of the oscillatory circuit, and the subsequent exchange of this energy with the molecular system of the liquid, leads to a change in the physical and physical and chemical parameters of the fuel. Ultimately, the electrophysical effect results in a change in the rate constant of the chemical reaction $K_{f}$ due to the Arenius law due to the change in the activation energy $E_{a}$, which in turn affects the speed and completeness of the combustion:

$$
K_{f}=k_{o} \sqrt{T} e^{\frac{-E_{a}}{R T}}
$$

where $k_{o}$ is the coefficient, depending on molar masses of matter and sizes of reacting molecules; $e^{\frac{-E_{a}}{R T}}$ is the exponential Arenius function.

The most important indicator of the quality of diesel fuels is the tendency to ignite when in contact with air. The flammability of fuels is estimated by the cetane number on the value of which, in turn, determines the duration of the ignition delay period $\tau_{i}$. The discrepancies in determining the cetane number, according to the flash coincidence method, of the initial fuel did not differ from the arithmetic mean of the compared results by more than \pm 1.4 cetane units.

The tests have shown that after the electrophysical impact on diesel fuel, the cetane number increases by $2 \ldots$ 2.5 units. An increase in the cetane number indicates an increase in the flammability of hydrocarbon fuels, a decrease in the ignition delay $\tau_{i}$, which is determined by the formula:

$$
\tau_{i}=\frac{\text { const }}{A_{\mathrm{cM}}} P^{-v} e^{\frac{E_{a}}{R T}}
$$

where $A_{\mathrm{cm}}$ is a factor depending on the composition of the reacting mixture; $v$ is the total order of the branching reaction; $P$ is the air pressure in the cylinder; $E_{a}$ is the activation energy; $T$ is the temperature of the reacting mixture.

The rate of chemical reaction of combustion of fuel is proportional to the product of the concentration of reactants at the same time:

$$
W_{f}=\frac{d C_{f}}{d \tau}=k_{f} C_{f}^{v_{f}} C_{k}^{v_{k}}
$$

where $k_{\mathrm{f}}$ is the constant of the reaction rate; $C_{\mathrm{f}}, C_{\mathrm{k}}$ is the concentration of fuel and oxygen respectively; $v_{f}, v_{k}$ are the reaction orders for fuel and oxygen, respectively.

Consequently, when the energy of activation $E_{a}$ decreases, as a result of the electrophysical influence on the energy of the intermolecular interaction between the fuel and oxygen molecules, the rate of the burning reaction of the fuel $W_{f}$ will increase. Increasing the oxidation reaction rate $W_{f}$ in the main combustion phase is one of the requirements of improving the economy and contributes to the increase of combustion completeness. Improvement of combustion processes in the previous phases leads to a reduction of the burning phase.

Completeness of combustion and tendency to carbonization is the main performance characteristics of hydrocarbon fuels, which are of great practical importance in improving the efficiency and engine life. The main emissions that pollute the atmosphere in exhaust gases of engines are carbon monoxide $(C O)$, unburned hydrocarbons $\left(C_{n} H_{m}\right)$, nitrogen oxides $\left(N O_{x}\right)$ and smoke with solid soot particles, which reduces the transparency of the atmosphere and contains carcinogenic substances. Since the specific emission is equal to the quantity emitted during the combustion of $1 \mathrm{~kg}$ of fuel, substance $\mathrm{j}$, the emissions of $\mathrm{CO}$ and $\mathrm{CnHm}$ are determined by the value of the coefficient of completeness of combustion of fuel.

About the tendency to carbon formation is judged by the amount of soot, which is deposited on the walls of the combustion chambers, while the aerodynamics of the flow is disturbed, local overheating, distortion and cracking of the flame tubes occur. The carbon particles carried away by the gas flow cause erosion of metal surfaces. When a deposit is deposited on the injectors, the shape of the sprayed jet changes, the efficiency of fuel combustion decreases [4].

The use of qualifying methods for testing fuels and lubricants in conjunction with physico-chemical research methods, with minimal consumption of funds and liquid samples tested, makes it possible to reliably evaluate their performance properties, that is, using qualification methods, it is possible to accelerate the assessment of the performance properties of fuels and lubricants. optimal mode and efficiency of the electrophysical method of exposure [ 5].

\section{FEATURES OF QUALITY ASSESSMENT}

Completeness of combustion and tendency to carbonization is the main performance characteristics of hydrocarbon fuels, which are of great practical importance in improving the efficiency and engine life.

About the tendency to carbon formation is judged by the amount of soot, which is deposited on the walls of the combustion chambers, while the aerodynamics of the flow is disturbed, local overheating, distortion and cracking of the flame tubes occur. The carbon particles carried away by the gas flow cause erosion of metal surfaces. When a deposit is deposited on the injectors, the shape of the sprayed jet changes, the efficiency of fuel combustion decreases [5].

The use of qualification test methods for combustivelubricating materials in conjunction with physico-chemical research methods, with minimal consumption of funds and test samples of the liquid, makes it possible to reliably evaluate their performance properties, i.e. using qualification methods, it is possible to speed up the assessment of the performance properties of fuels and lubricants, to identify the optimal mode and efficiency of the electrophysical method of exposure [2, 4].

\section{DESCRIPTION OF THE EXPERIMEN}

Conducted research to determine the effect of electrophysical effects on the characteristics of the combustion of hydrocarbon fuels in a small installation. The method makes it possible to give a comparative assessment of the completeness of combustion and carbonization of fuels for engines with a small amount of fuel spent on 
testing. Aviation fuel was selected as the object under study. The test of the control sample of the fuel and the sample subjected to electrophysical influence was carried out on the same stand.

The combustion chamber is made according to the type of combustion chamber of a turbojet engine. The flame tube of the small-sized chamber is collapsible. The temperature of the exhaust gases was recorded using a potentiometer (11 measurement points around the perimeter of the nozzle of the combustion chamber). Directly in front of the combustion chamber, a device for electrophysical influence on fuel is included in the stand system.

Conducted research to determine the effect of electrophysical effects on the characteristics of the combustion of hydrocarbon fuels in a small installation. The method makes it possible to give a comparative assessment of the completeness of combustion and carbonization of fuels for engines with a small amount of fuel spent on testing. As the test object was selected fuel. The test of the control sample of the fuel and the sample subjected to electrophysical influence was carried out on the same stand.

The possibility of carbon formation and the appearance of tar, carbonaceous and coke-like deposits in engines is closely related to the completeness of fuel combustion. Before evaluating fuels for a tendency to carbon formation, the middle part of the flame tube of a small-sized combustion chamber was cleaned of carbon, dried at $420 \mathrm{~K}$, weighed, then the chambers were mounted on a stand.

The tests were carried out under constant experimental conditions for 1 hour. Then the chamber was removed, the middle part of the flame tube with the carbon deposited in it was disassembled, after removal of soluble precipitates and drying, weighed. The difference in weighings before and after the test determined the amount of soot.

Experiments have shown that with control fuel, the amount of soot was $\mathrm{x}_{1}=5.30 ; 5.29 ; 5.5 ; 5.45 ; 5.3 ; 5.50$; $5.49 ; 5.5\{\mathrm{~g}\}$, and after electrophysical exposure $\mathrm{x}_{2}{ }^{\mathrm{EF}}=$ $4.10 ; 4.09 ; 4.19 ; 4.15 ; 4.20 ; 4.19 ; 4.16 ; 4.20\{\mathrm{~g}\}$.

\section{EVALUATION OF UNCERTAIN}

In the work for the analysis of the process of testing the completeness of carbon dioxide combustion with the use of the device with electrophysical influence, the calculation of the combine uncertainty for the indicators of the quality of the engine performance indicators, namely, indicators of the formation of carbon in the combustion chamber [2].

The arithmetic mean of a number of observations according to the described experiment for a reference fuel is:

$$
\begin{aligned}
\bar{X}=\frac{1}{n} \sum_{1}^{n} x_{i}= & \frac{1}{8}(5,30+5,29+5,5+5,45+5,3 \\
& +5,50+5,49+5,5)=5,42 \mathrm{~g}
\end{aligned}
$$

For fuel after electro-physical impact :

$$
\begin{aligned}
\overline{X^{E F}}=\frac{1}{n} \sum_{1}^{n} x_{i}^{E F} & =\frac{1}{8}(4,10+4,09+4,19+4,15+4,20 \\
& +4,19+4,16+4,20)=4,16 \mathrm{~g}
\end{aligned}
$$

Standard uncertainty for type A of measuring result [4]:

$$
u_{A}\left(x_{i}\right)=\sqrt{\frac{1}{n(n-1)} \sum_{i=1}^{n}\left(x_{i}-\bar{x}\right)^{2}}
$$

Standard uncertainty for type A for reference fuel:

$$
\begin{aligned}
u_{A}\left(x_{i}\right)^{2}=\frac{1}{8(8-} & =\left((5,30-5,42)^{2}+(5,29-5,42)^{2}\right. \\
& +(5,5-5,42)^{2}+(5,45-5,42)^{2} \\
& +(5,3-5,42)^{2}+(5,50-5,42)^{2} \\
& \left.+(5,49-5,42)^{2}+(5,5-5,42)^{2}\right) \\
& =0,00126
\end{aligned}
$$

$$
u_{A}\left(x_{i}\right)=\sqrt{\frac{1}{n(n-1)} \sum_{i=1}^{n}\left(x_{i}-\bar{x}\right)^{2}}=\sqrt{0,0126}=0,0355 .
$$

Standard uncertainty for type A for fuel after electrophysical influence:

$$
\begin{gathered}
u_{A}\left(x_{i}^{E F}\right)^{2}=\frac{1}{8(8-1)}\left((4,10-4,16)^{2}+(4,09-4,16)^{2}\right. \\
+(4,19-4,16)^{2}+(4,15-4,16)^{2} \\
+(4,20-4,16)^{2}+(4,19-4,16)^{2} \\
\left.+(4,16-4,16)^{2}+(4,20-4,16)^{2}\right) \\
=0,000243
\end{gathered}
$$

Standard uncertainty of type B for $x_{i}$ :

$$
\begin{gathered}
u_{B}\left(x_{i}\right)=\frac{b_{i}}{\sqrt{3}} \\
u_{B}\left(b_{i}\right)=\frac{0,01}{\sqrt{3}}=0,0058
\end{gathered}
$$

Calculation of the contribution of the uncertainty of the input value to the uncertainty of the measured value.

Model equation:

$$
\rho=\frac{m}{V}
$$

The combine standard uncertainty $u_{c}(y)$ was defined as the product of the uncertainty of the input value $u\left(x_{i}\right)$ for the sensitivity coefficient $c_{i}$ :

$$
\begin{gathered}
u_{c}(y)=\sqrt{c^{2}(V) * u^{2}{ }_{A}\left(V_{i}\right)+c^{2}(m) * u^{2}(m)} \\
c=\frac{\partial y}{\partial x} \\
\rightarrow \quad c(V)=\frac{\partial \rho}{\partial V}=-\frac{m}{V^{2}}=-\frac{4,16}{2000^{2}}=-0,034 \mathrm{~g} / m l^{2} .
\end{gathered}
$$

The contribution of the uncertainty of the volume to the uncertainty of the measurements is equal:

$$
u_{1}=c_{1}(V) \cdot u_{B}(V)=-0,034 \cdot \frac{0,05}{\sqrt{3}}=-9,7 \cdot 10^{-5} \mathrm{~g}
$$


The weight sensitivity is equal:

$$
c(m)=\frac{\partial \rho}{\partial m}=\frac{1}{V}=\frac{1}{2000}=0,0005 m l^{-1} .
$$

The contribution of the measurement uncertainty of the mass c without the electrophysical effects of type A to the measurement uncertainty is:

$$
\begin{gathered}
u_{2}=c_{1}(m) \cdot u_{A}\left(x_{i}\right)=0,0005 \cdot 0,0355 \\
=0,000001775 \mathrm{~g}
\end{gathered}
$$

The contribution of the uncertainty of mass measurements of type B to the uncertainty of measurements is equal to:

$$
u_{3}=c_{1}(m) \cdot u_{B}\left(x_{i}\right)=0,0005 \cdot 0,0058=0,0000029 g
$$

Consequently, the combine standard uncertainty [4] without electrophysical influence equals:

$$
\begin{gathered}
u_{c}(y)= \\
\sqrt{c^{2}(V) * u^{2}(V)+c^{2}(m) * u^{2}{ }_{A}\left(x_{i}\right)+c_{1}(m) \cdot u_{B}\left(x_{i}\right)}= \\
\sqrt{(-0,000097)^{2}+0,000001775^{2}+0,0000029^{2}} \\
=9,88 \cdot 10^{-5} g
\end{gathered}
$$

Coefficient of coverage at $\mathrm{P}=0,95 \rightarrow k=2$

Extended uncertainty equals:

$$
\begin{gathered}
U=k * u_{c} ; \\
U=9,88 \cdot 10^{-5} * 2=0,0001975 g
\end{gathered}
$$

As a result of the imperfection of the measurement of the weight of carbon nanotubes without electrophysical influence, the result can be represented as:

$$
m=(5,42 \pm 0,00020) g, P=0,95 .
$$

The contribution of the uncertainty of mass measurements in an electrophysical influence of type A to the uncertainty of measurements is:

$$
\begin{gathered}
u_{2}=c_{1}(m) \cdot u_{A}\left(x_{i}^{E F}\right)=0,0005 \cdot 0,01558 \\
=0,00000779 \mathrm{~g}
\end{gathered}
$$

The contribution of the uncertainty of measurements of the mass of type B to the uncertainty of measurements is equal to:

$$
u_{3}=c_{1}(m) \cdot u_{B}\left(x_{i}\right)=0,0005 \cdot 0,0058=0,0000029 g
$$

Consequently, the combine standard uncertainty with an electrophysical effect is equal to:

$$
\begin{gathered}
u_{c}(y)^{E F}= \\
=\sqrt{c^{2}(m) * u^{2}{ }_{A}\left(x_{i}{ }^{E F}\right)+c^{2}(V) * u^{2}(V)+c_{1}(m) \cdot u_{B}\left(x_{i}\right)} \\
=\sqrt{(-0,000097)^{2}+0,00000779^{2}+0,0000029^{2}} \\
=\quad=9,7 \cdot 10^{-5} g
\end{gathered}
$$

Coefficient of coverage at $\mathrm{P}=0,95 \rightarrow k=2$

Extended uncertainty with electrophysical influence:

$$
\begin{gathered}
U^{E F}=k * u_{c} ; \\
U^{E F}=9,7 \cdot 10^{-5} * 2=0,000195 g
\end{gathered}
$$

The result of the measurement with an electrophysical effect has the form:

$$
m^{E F}=(4,16 \pm 0,00020) g, \mathrm{P}=0,95 .
$$

An analysis of the experimental data on the formation of the formation in the combustion chamber of the thermal motors and the calculation of the uncertainty of the influence of factors in the evaluation of the quality index of the operation of these engines gives an opportunity to draw a conclusion. Electrophysical action on hydrocarbon fuel reduces deposits of carbon on the walls of the combustion chamber by $23 \%$. Changes in the physico-chemical, operational and environmental properties of hydrocarbon fuels are possible with electrophysical effects. This method of influencing the physicochemical properties of hydrocarbon fuels reduces fuel consumption and reduces the toxicity of exhaust gases under operating conditions.

\section{CONCLUSION}

In the work, an analysis of the results of engine tests was conducted. The results of theoretical and experimental studies have shown the effectiveness of the application of electrophysical effects on hydrocarbon fuels, which leads to a reduction in the mass of carbon in the combustion chamber, that is to increase the environmental indicators as indicators of the quality of operation of internal combustion engines. The total uncertainty was calculated for the use of control fuel and fuel after electrophysical influence. The proposed studies form the basis for developing recommendations for reducing uncertainty in the operation of fuel engines. Especially when it comes to such exhaustive energy sources as gasoline [8].

\section{REFERENCES}

[1] Yu. M. Tereschenko, S. L. Maksimov I. V. Morozova,. Change of heat engine performance by electro-physical influence on fuel // The Advanced Science Journal. - ISSUE 02 / VOLUME 2016 /ISSN 2219-746X. - P. 45-49.

[2] Trofimov, I. Study of antiwear properties of ts-1 jet fuel treated with electric field / I.L. Trofimov, N.N. Zakharchuk // Systems and means of motor transport (selected problems), by Politechnika Rzeszowska. - Rzeszow, Poland. - p. 295-301.

[3] I. V. Morozova, V.I. Morozova . Influence on the energy efficiency of hydrocarbon fuels // Nanostructured materials for the delection of CBRN.-2017. P. 319-330. DOI: 10.1007 / 978-94-024-1304-5 24

[4] Aleksandrov E. E.,. Kravec I. A, Lysikov E. P., Solovyov O. V., Tropina A. A.. Increase of resource the technical systems by the use of the electric and magnetic fields. NTU „KHPI”. P. 544. (2006).

[5] I. V. Morozova Increase of efficiency of control of dielectric properties of petroleum products // Electronics and control systems. 2010..№ 3(25). pp. 40-43

[6] O.Ivanets, V.Morozov, I.Morozova. Analyze the dynamic electrically infused into the aquatic environment// Oil and gas industry. 2012 №1. p. 64-66

[7] ISO/IEC Guide 98-1:2009 1:2009(JCGM/WG1/104) Uncertainty of measurement Part 1: Introduction to the expression of uncertainty in measurement.

Trofimov, I. L. The increase of tribotechnical properties of fuels and oils by the action of the electric field. Questions of chemistry and chemical technologists. №3/2010. - P. 132-137. 